\title{
Can life be engineered? Epistemological roots and blind spots of Synthetic Biology
}

\author{
Thomas Heams ${ }^{1,2, a}$ \\ ${ }^{1}$ INRA, UMR 1313, Génétique Animale et Biologie Intégrative, Domaine de Vilvert, \\ 78352 Jouy-en-Josas Cedex, France \\ ${ }^{2}$ AgroParisTech, Département Sciences de la Vie et Santé, 16 rue Claude Bernard, 75231 Paris \\ Cedex 05, France
}

\begin{abstract}
Synthetic Biology is the latest attempt in experimental biology to reach the long lasting goal of mastering processes of life by engineering them. This emergent discipline results from the novel convergence of biology and concept and tools from other fields such as computing and engineering sciences. It relies on rational design of bioparts, modules, or organisms, as opposed to the tinkering methods provided so far by the even most sophisticated biotechnologies. Such an approach could have major consequences, for both applied and fundamental research. But this appealing narrative may obscure important epistemological issues, some of them being rooted in old misconceptions or shortcomings in biology. By focusing mainly on the mechanistic dimension of living beings, Synthetic Biology partially recycle ancient debates and could miss the opportunity to provide an integrative account of what makes life actually specific in the natural world. A first insight into a critical reassessment of some of the goals, the lexicon, and the theoretical foundations of Synthetic Biology is proposed, as other natural dimensions of the biological world are highlighted. Taken as a whole, these considerations challenge several core concepts of the discipline, but may help to redefine some of its strategies and overcome some major hurdles.
\end{abstract}

\section{Introduction}

Synthetic Biology has recently rephrased the ancient project of engineering life. Since humans have been able to transform their environment, mastering the processes of life has been a constant goal, as well as a frontier which has inspired transgressive fears or dreams that turned into universal myths such as the Promethean legend and its modern avatar, Mary Shelley's novel Frankenstein published at the dawn of the modern industrial era in Europe [1]. Both question human individual freedom and responsibility, as if understanding the nature of the threshold between life an non-life, and even trying to trespass by building organisms from inorganic or dead parts, was a decisive contribution to the very definition of liberty, encompassing here the specific issue of freedom of research. Far from mythologies, modern science has taken this question over by organizing knowledge about living beings following a process of deconstruction whereby organisms have been described in ever smaller parts, that

\footnotetext{
${ }^{\text {a}}$ Corresponding author: thomas .heams@agroparistech.fr
}

This is an Open Access article distributed under the terms of the Creative Commons Attribution License 4.0, which permits unrestricted use, distribution, and reproduction in any medium, provided the original work is properly cited. 
is organs, tissues, cells, then genes. The theoretical assumption underlying this reduction, made possible by correlative progresses in technology, was that a day would come were a rebuilding process would be at hand. To some extent, this framework has proven relevant, yet not so far in terms of experimental biology: computer simulations can indeed already model cells, taking into account their components, and provide accuratein silico predictions of variations in e-cell behavior if e-genes are switched off or if new e-nutrients are provided [2]. Is it only a matter of time for in vivo approaches to reach the same point? That is one of the major claims put forward by the new discipline that is Synthetic Biology. The extensive amount of knowledge stored in databases, combined to new biotechnological tools, rational design strategies and the exponential power of computers would make this goal achievable [3]. As a consequence, biology would switch from description to transformation, more than it has ever done before, with major fundamental consequences (such as a deeper understanding of what biological life actually is) and applied ones as well (such has entering living organisms in industrial processes). But turning biological life into technology is a narrative that deserves careful scrutiny, which is the purpose of the present paper. Following some clarifications regarding the definitions and the research programs of Synthetic Biology, a critical reassessment of its intellectual foundations will be proposed. This will substantiate an analysis of several pitfalls in which Synthetic Biology may be trapped, and provide some epistemological tools to reconfigure its strategies and agenda, in line with more integrative accounts of biological life. Finally, some new trends in Synthetic Biology will be highlighted, which open new roads and tend to show that this approach has strong internal resources to reach promising milestones for fundamental and applied life sciences.

\section{Under the umbrella: Synthetic biology in the lab}

Synthetic Biology can be defined as a recent multidisciplinary field of research that aims at using rational design strategies to study and transform biological objects, from biomolecules to organisms, by the combined use of concepts and tools from life sciences and their surroundings (biology, biochemistry, biotechnologies) and from other disciplines, among which informatics, electronics, nanotechnologies and engineering sciences [4]. This results in a large, sometime heterogeneous, research program with both fundamental and applied dimensions. Relying on themselves recent, and often technology-driven disciplines, Synthetic Biology promises to take each of these dimensions to a new level, by addressing theoretical issues such as the definition of life itself with new experimental opportunities (e.g. by developing protocells or by streamlining genomes, see below) or by looking for novel biotechnological answers to industrial, sanitary or environmental challenges (by engineering cells or organisms). Thus it is a very large and appealing umbrella concept, all the more that the Synthetic Biology's attractiveness relies on these pioneering perspectives and claims to challenge traditional boundaries in academic sciences. Noteworthily, this promise-oriented dimension is rooted in the proto-history of the discipline [5]: during the twentieth century, the very term "Synthetic Biology" sporadically surfaced at key tipping points in the history of biology that raised comparable hopes of the transition from description to transformation. Among famous forerunners, Stéphane Leduc coined the term as the title of his essay La Biologie Synthétique in 1912 [6], in the mendelian laws rediscovery era, and Wacław Szybalski reintroduced it in 1974 [7] when genetic engineering started to take off thanks to the recent availability of restriction enzymes. Obviously, these two early mentions do not fully fit with the modern meaning of Synthetic Biology as defined by the founding first congress of the discipline at the MIT in $2004^{1}$, but they nonetheless underline its ancient foundations and expectations. Beyond these common definitional and historical aspects, Synthetic Biology is itself, in its contemporary sense, composed of different, yet connected and complementary, subfields. The tentative cartography developed below follows and

\footnotetext{
${ }^{1}$ http://syntheticbiology.org/Synthetic_Biology_1.0.html.
} 


\section{ORIGINS}

summarizes a typology proposed by in 2008 [8] that delineates three constitutive areas in Synthetic Biology: genome engineering, DNA-based device construction, and protocell research.

Genome engineering consists in using genomes as a template for extensive modifications such as but not exclusively- complete chemical synthesis, complete genome replacement, chromosome editing, shuffling and/or streamlining. The more these techniques are combined, the more the outcomes are considered as experimental milestones (see for example [9, 10]). Genome engineering, grounded in previous studies on minimal gene sets thanks to comparative genomics [11], serves as a good example of the pluripotentiality of Synthetic Biology. Indeed, such sustainable lineages of organisms with massively reduced genomes can be experimental clues for putative ancient supposedly "primitive" forms of life, as well as bases for the understanding of extensive adaptative reductions in case of extreme forms of symbioses such as endosymbiosis (intracellular bacteria). On the other hand, it is sometimes claimed that they could provide standardized biological chassis [12], that is minimal unicellular organisms upon which subsequent rationally deviced genetic modules could be further incorporated on demand, for various goals, from drug delivery to bio-remediation.

DNA-based device construction is based, to a large extant, on the principles of genetic engineering and transgenesis, and aims at developing strains of unicellular organisms embedding rationally conceived genetic modules, whether they are made of genes or of "parts", like biobricks [13], which are themselves small DNA modules, i.e. standardized units of functions (e.g. coding sequences regulatory elements and shared linker sequences). Proponents of rational design, inspired from engineering science, put this method forward to overcome the many limitations of previous transformation methods like artificial selection or genetic engineering, ruled by unpredictability [14]: natural and artificial selection indeed rely on random events such as mutation occurrence, allelic partition and recombination during meiosis, and gametes mating during fertilization. Even genetic engineering have long been based on random integration of transgenes. With rational design and standardization, Synthetic Biology is supposed to bypass most of this "black box"syndrome, and implement surgical precision in biological modification methods. DNA-based devices can result in complex compounds such as drugs produced from long cascades of gene expression, and promises of such research are large, whether they are applied (biofuel production, biosensing, remediation) [15], or fundamental, such as generating new intracellular structures with concatened RNAs [16]. Compared to classical genetic engineering, Synthetic Biology approaches add several novelty elements in the process among which (i) intentional design of modules, based on preexisting knowledge from other organisms, and/or on engineering principles from other disciplines, like electric engineering [17], and (ii) integration of such modules in pre-existent organisms. As modules get complex, successful integration becomes a major experimental challenge, for size reasons, but even more because of the high degree of unexpected perturbations they can trigger is the host, which can rise exponentially with the number of coding sequences introduced due to the high connectivity naturally existing in gene networks. Additionally (iii) the above mentioned standardization process, coupled with an open source philosophy at the parts level, opens the the door to novel modes of scientific empowerment by social practices of biology outside the academic or companies laboratories, such as bio-hacking or DIY biology movements [18].

DNA-based device construction is often the most visible part of Synthetic Biology as a whole. At the pedagogical level, the ever growing success of the flagship iGEM competition [13], where student teams from various countries present proof-of-principles of modified organisms using this biobrick registry of part $^{2}$, has triggered collaborative innovations in research and teaching among participating universities or research centers. At the pre-industrial research level, this approach has already produced several results, such as the synthesis of a precursor of the artemisinic acid in yeast (originally produced in the plant Artemisa аппиа) [19] or the hydrocortisone [20]. The perspectives theoretically opened by the

2 http://parts.igem.org/Main_Page 
standardization of parts, and by the rational conception of modules, are obviously appealing for the industry sector, whether it is a the engineering level or at the intellectual property level, in contrast with classical biotechnologies, that have also yielded results but still have a hard time overcoming the fundamental behavioral unpredictability of living organisms. DNA-based device construction extends in xenobiological approaches that is the design, synthesis, and incorporation of non natural nucleotides in existing genomes and organisms [21]. Extensive biochemical repertoires of modified nucleotides exist that could in theory replace or complement the four universal nucleotides of DNA [22], the challenge being to actually perform the in vitro incorporation of such bases in stable way over cell divisions [23], with prospects to turn them into functional sequences. In parallel, experiments of rewriting and expansion of the almost universal Genetic Code, that is the correspondence between nucleic-acid and amino-acid sequences are on their way and have already proven that other directions for the living world are possible [24]. Combining xenobiology and genetic code expansion would further diversify these theoretical and experimental explorations. Here again, such research has both fundamental and applied implications, from testing alternative but plausible exotic forms of life [25], to bio-safety by making microorganisms dependent on non-natural components, thus confined in the very laboratory where they have been developed, or the zone they are expected to be active in [26]. Nonetheless, whether these xeno-organisms can reverse or reject these modifications remains an open issue, as a consequence of them being a biological lineage with its own intrinsic evolutionary nature.

Protocell research relates to experimental studies aiming at understanding the fundamental transitions from non-living to living matter. As such, they are strongly connected with chemistry and biochemistry, including the study of infrabiological entities such as association of lipid or proteic vesicles and simple autocatalytic molecules. Their combined evolution and mutual dependance could lead to truly living entities, that is systems that would unequivocally display simple metabolism, selfreplication, heredity and evolvability features ${ }^{3}$ [27]. At first sight, such protocells would look far much simpler than streamlined bacteria, let alone the engineered ones from the two above mentioned other areas of Synthetic Biology. Indeed, protocell studies provide the best experimental approximations of what could actually have been the earliest forms of life on Earth (at least in the conceptual dominant framework assuming that life evolved from simple to complex forms, a common-sense hypothesis that however deserve careful scrutiny [28]) even if the historical ones are obviously lost for ever. But behind this apparent simplicity, such a complete transition remain elusive, for no one has yet overcome all the hurdles that could generate a simple life form from its chemical components only. It is noteworthy that this transition from inorganic matter to organic life -as we know it- still resists experimental reproduction, when the other approaches in Synthetic Biology, with seemingly more complex structures, already have started to deliver. Whether this originates in temporal technical limitations of protocell research or in theoretical misconceptions is discussed and will be partially addressed below. Protocell research is mostly a fundamental approach, that have close connections with Origins of Life studies, even if the two disciplines only partially overlap. It is also of interest, as is xenobiology, when considering Exobiology, that is the study of potential life forms in the Universe [29], or putative shadow biospheres on Earth [30]. Experimental protocell research enables to study to what extent non canonical types of membranes, of informational molecules, and combination of such could exist elsewhere our planet, therefore opening questions about how to detect such life forms, or traces, if they exist somewhere. As a result, Origins of Life studies, Exobiology and Protocell research, each having their own specificity, nonetheless delineate an "epistemological triangle" at the center of which the fundamental nature of biological life is questioned by science. But aside this fundamental aspects, protocell research could also open applied opportunities, such as the design of novel infrabiological

\footnotetext{
${ }^{3}$ See note $g$ for a critical reassessment of such list.
} 


\section{ORIGINS}

vectors, neither bacterial nor viral, but with partial combined characteristics of life, that could unlock new therapeutic solutions, e.g. in targeted drug delivery [31].

Thus, the emerging field that is Synthetic Biology is one of many challenges, at every scale, from non-living/living transition, to chemically synthesized genomes, and each step in between. The farther the finishing line from the start, the higher this challenge is. By demonstrating that unexpected life forms, or biological products, can be developed in the laboratory, Synthetic Biology also challenges the long lasting distinction between artificial and natural (here, biological) objects. In this regard, it leads to apparent paradoxical epistemological situations: bacteria which genome have been experimentally reduced, and therefore have been extensively engineered from their original form, could well be, in consequence, arguably labelled as "artificial" or "synthetic"[9], and in the very same time be considered of major interest for studying the minimal requirements for life, thus contributing to its very definition. In the same vein, Ichells composed of metal, are tools to investigate fundamental aspects and limitations of life [32]. This paradox fades when synthetic biologists point out that precisely, they do not consider the study and transformation of living beings as two parallel activities but, on the contrary, as the two sides of the same coin: they seek to understand life by transforming it or even to create it, as claimed by Richard Feynmann in his famous quote that became a quasi-official mantra of Synthetic Biology "What I cannot create, I do not understand"4 . This logic being fruitful in many non biological disciplines, it is tempting to implement it, now that technologies seem to allow it, in this long resisting field. But there are many reasons why this reasoning is not straightforward, and could explain some limitations in the nascent field of Synthetic Biology. And before addressing them, an historical detour might be necessary.

\section{Under the carpet: The roots of mechanistic depiction of life and their consequences}

From an anthropological perspective, Synthetic Biology is one among the most recent attempts in the long series of human activities aiming at rationally transforming biological systems. Agriculture, domestication, artificial selection of crops and livestock, and medicine relate to this tradition so ancient and universal that conscientiously transforming the living world could be considered as one of the defining characteristics of our species. So the first questions at stake are (i) to provide an accurate definition of what "understanding and/by transforming life" means for synthetic biologists, and (ii) to assess how novel this vision is.

From an historical perspective, understanding the living world has always resulted from the study two types of its dynamics, internal (how organisms, and cells, behave, react, and adapt) and later with ecology and evolutionary sciences, external (how organisms interacts and evolve). In the study of internal dynamics, of which Synthetic Biology is the last avatar, there has been a constant tradition of focusing on mechanistic depictions of living systems. Accounting for machines as an approximation of biological systems is deeply rooted in the history of life sciences in the Modern era, including such milestones as the depiction of systemic circulation of blood by William Harvey, and the animal-machine dualistic theory proposed by René Descartes [33] then Nicolas Malebranche, and later transposed to the human species by Julien Offray de la Mettrie [34]. This comparison between organisms and machines was strong enough to fit even opposite visions of life, like de la Mettrie's materialist standpoint, as well as creationist depictions of living world championed a few decades later by the leading figure of the fixist pre-darwinian era, William Paley, famously comparing living beings to watches as a proof of the necessary existence of a divine watchmaker [35].

The parallelism between organisms and machines, the latest being human artefacts, remained attractive until today, so that artificiality has been tacitly embedded in biological systems. Organisms and

\footnotetext{
${ }^{4}$ http://archives.caltech.edu/pictures/1.10-29.jpg
} 
cells have continuously been compared to actual machines, from XVIII ${ }^{\circ}$ century automatons to modern days computers. The advent of molecular genetics, contemporary to the birth of computer sciences after World War II with which it shares common intellectual foundations [36] and framework underlined by a common lexicon (e.g., program, sequence, data, code, signals, hacking), raised this parallelism to an unprecedented level and substantiated a reinforced reductionist depiction of life, describing organism or cell behaviors as the result, even complex, of their fine-tuned parts. By implementing standardization and rational design in biotechnology, Synthetic Biology research has gone even farther in this direction $[37,38]$. This mechanistic rationalization of "life" seems not only to fit with standardized and therefore industrial approaches, but also to pave the way for the annexation of the living world by the dynamics of the free-market economy. Indeed, one can notice that living beings have a long lasting but contrasted relationship with the history of capitalism. From the enclosure movement that restricted of the common access to land for livestock at the dawn of British Agricultural Revolution, to the most modern forms of biopower [39], living beings have been key elements of this economic system (itself in perpetual need of new market opportunities), either by providing inputs and labour force, or on the other hand, by resisting its standardization needs. In this regard, Synthetic Biology promises are all but neutral: if biological life can be atomized into modules, whether it is under a copyright or an open source philosophy, it can cope with the rules of consumers society, let alone fuel libertarian fantasies of transhumanist convergence between man and machine. Would such premises be right, Synthetic Biology would then be a potential technical revolution, a possible change of paradigm, but first of all an actual ideology.

Thus, establishing a sort of parenthood between living systems and machines raises several epistemological issues. First, as mentioned above, this reduction of living systems to their mechanistic dimension is far from novel and gives no guarantee, even if rephrased in biotechnological terms, of generating per se a convincing scientific explanation of life (i.e. from a methodologically naturalistic perspective). It is potentially compatible with other theories such as dualism or creationism, which are out of the scope of scientific naturalistic explanations, for they presuppose supernatural forces at work. Furthermore, this "special relationship" leads to decipher biological order (which is the major explanandum of biology) by relying on pre-existent order at smaller scales, that is to depict biological systems as fine-tuned systems. In a modern evolutionary perspective, this amounts to consider that most evolutionary adaptations consist in precise gene regulation networks enabling relevant reactions to environmental variations or to internal signalling pathways. This lead to another paradoxical situation where order is both the explanans and the explanandum. This tautology, yet partially explained by the cybernetic nature of most biological reactions (where retroaction loops can turn consequences into causes) [40], is nonetheless an epistemological blind spot of the reductionist-mechanistic approaches of life. When Synthetic Biology claims to understand life by transforming it, it submissively fits this framework, where the default state is order of which experimental distortions are expected to reveal the very nature of the complex mechanisms at play in the "living machine". By this standard, Synthetic Biology, itself a discipline heavily powered by computer sciences, is strongly self-consistent. But would this consistency persist if the very bases of this paradigm were challenged? Such a question could look presumptuous, considering the tremendous amount of experimental results obtained by such reductionist frameworks in molecular biology. In first approximation, gene regulation networks, enzymatic reactions, metabolic pathways and stereospecificity features have a lot in common with mechanics. But precisely, because mechanisms exist far beyond the living world, this resemblance, as it will be discussed below, does not accurately capture the very specificity of living entities. Moreover, every laboratory biologist experiences the limitations of such comparison. Thus, a critical assessment of the weaknesses of these premises could well be necessary, and even urgent, but is seldom addressed. This current lack is puzzling, given that Synthetic Biology fosters a high degree of self-reflexivity, even if by the means of a sophisticated use of interlaced facts and narration, and sometimes of blurred lines between the high level of expectations, the substantial number of proof-of principles, and the smaller number of actual published breakthroughs. But precisely, the content of this narrative may explain 


\section{ORIGINS}

why such a lack remains. Discourses on the promises of the discipline focus on the type of hybrid entities Synthetic Biology could produce, and what interactions such hybrids could engage with the natural world, taking for granted that it has a high efficiency potential, for better or worse. In doing so, they bridge rational techno-scientific challenges with mythical representations of transgression in a combination that contributes to the fascination power of the discipline, on the public or on the scientists themselves. Here, Synthetic Biology is at the same time a methodological resource for delineating the core definition of biological life, and by construction a boundary discipline: crossing limits is one of its constitutive aspects, which implies subtle ethical debates. As a result, social scientists embedded in Synthetic Biology rather study the mixed perceptions of these possibilities and the consecutive polarization between fears and hopes [41]. In this context, there is little audience for divergent concerns about the fragility of Synthetic Biology premises.

\section{An elephant in the room: Why life cannot be engineered}

The current state of Synthetic Biology is contrasted. This scientific community is small but visible, enthusiast and dynamic, present in the traditional and social media spheres, and proactive in interfacing public and private research. More than 3000 research articles have been published since 2004, attendees number in dedicated conferences and meetings keep growing, and the discipline arouses the attention of many government agencies and NGO worldwide. On the other hand, it has not so far been as revolutionary as one could reasonably have expect it to be. To put it bluntly, to date (i) living entities have not been build from scratch, (ii) rational design and standardization of part have not outcompeted previous methods of biotechnology, and (iii) minimal bacterial chassis have not provided many therapeutic or bio-remediation tools. Whether this is due to the infancy of the discipline is an open question, and it would be pointless here to be judgmental or to dismiss the exciting roads that many synthetic biologists open. But looking at the facts, and considering that important companies recently started to refocus their previous investments and research in Synthetic Biology ${ }^{5}$, it follows that the current situation obviously deserves attention. Transfers from laboratory to business remains challenging and often elusive, and rational design strategies have not proven as disruptive as expected. By definition, exploratory multidisciplinary approaches and interface research projects do not all succeed in giving productive results. This will likely induce a global reassessment of the discipline's perimeter, and resized objectives more in line with reality than with early expectations.

In the light of this evolution, some of the very pillars of Synthetic Biology, are now extensively questioned, or at least redefined, among which rational design: it is a quite an unexpected turn of events to observe a renewal of interest for directed evolution methods (whether it is molecular or cellular), which are taking advantage of its fundamental randomness but in controlled chance/selection steps [42]. Even more so, some of the most spectacular methodological advances in genome engineering in the recent years come from methods based on accelerated evolution [43]. In a time where intentional design of part is slow to give results, darwinian dynamics, which directed evolution is inspired from, may not be so outdated after all for experimental biology.

But these first remarks do not constitute per se a convincing identification of the blind spots of Synthetic Biology. Would they be the only relevant critics, they could just highlight how young the discipline is, and some of the necessary clarifications to be done so that it may forsake its most provocative initial claims and actually start delivering. But it may well not be so: we claim here that by over focusing on the mechanistic dimension of life, Synthetic Biology put itself at risk, because meanwhile it does not confront, or not enough, to the other constitutive dimensions of biological life, that may sometimes even contradict the rationale behind life engineering. Yet, if all living organisms are

\footnotetext{
${ }^{5}$ http://www.bloomberg.com/news/2013-05-21/exxon-refocusing-algae-biofuels-program-after-100million-spend.html
} 
indeed material entities, they are not only mechanistic ones. Revisiting these other dimensions of life is crucial to address the blind spots of Synthetic Biology.

Before doing so, a point shall be made regarding the issue of complexity. Proponents of holistic or emergentist answers to the crisis of reductionism in biological thought, often state that the intrinsic complexity of biology, whereby "the whole is more than the sum of the parts", is the main reason why it resists mechanistic reduction [44]. Indeed, life has multilevel aspects, from molecules to organisms and even populations and ecosystems. The physiological (and evolutionary) transitions from one level to another significantly account for the difficulty in understanding the living world by reducing it to its parts (top-down knowledge), or to engineer from them (bottom-up construction). But we will not follow this path here for two main reasons. First, because the ontological status of these levels and transitions is far from obvious, and an convincing untangling of their objective and subjective dimensions is often lacking. Second and foremost, because when addressing core processes of life, focusing on its primordial complexity is irrelevant for a large part, for it would dismiss the fact that, from a structural and even functional point of view, some organisms are actually very simple compared to some sophisticated machines. It is hard to pretend, for example, that a mycoplasma, let alone a protocell, is more complex than a supercomputer, whatever the definition used for complexity. Reciprocally, so-called emergent properties, at the basis of the holistic standpoint, are hardly specific to biology. Instead, it might well be argued for example that complex softwares run by sophisticated computers generate emergent results that would make the computer and its outputs "more that the some of its part". It follows that emergent properties and complexity are non discriminant enough when exploring the specifics of biological life that are not captured by engineering. But other features exist that, taken together and combined, draw biological objects very far from machines, being complex or not.

Life, at least cellular life, is messy. As useful as the "program" metaphor (genetic, developmental) may have been for decades, it is now widely acknowledged that is is at best an approximation. Most text-book depictions of intracellular precise regulatory networks or feedback loops originate from average measurements from cell populations that do not account for their intercellular variability. When performed at the single cell level, they reveal the stochasticity of intracellular dynamics, which itself originates in molecular crowding, low number of regulatory molecules, and high connectivity of gene and protein networks [45]. Debates are ongoing about the status of this random variations, from them being a background noise or an actual biological parameter, but this unpredictability underlines that the observed macroscopic biological order (i.e. regulation at the organism level) does not necessarily rely on order at lower scales (e.g. fine tuned regulations at the cell level). Instead, it may take advantage of erratic exploratory individual cellular behaviors resulting in selectable, transient or lasting phenotypic cellular diversity $[46,47]$.

Life is collective, and mutually dependent. No individuals live alone, almost no species exists by itself. From endosymbionts to microbiomes, each organism or collective is connected to smaller or bigger ones by reciprocal, even if asymmetrical relationships. Within or between species, this collective dimension is one of the prerequisite for change by natural selection and for dynamic equilibria in ecosystems. Aside from the very specific issue of the Origins of Life, where there might have been once a truly single original cell (which is debated), dismissing this collective dimension is misleading, and defining the living world as a collection of individual and independent machines in which life would be mechanically insuflated is irrelevant. Life needs life to be life, that is a tautological feature that challenges our legitimate needs of categories, and drives living forms very far from objects that could be upgraded one by one.

Life is history. Stones, man-made machines and living beings obviously comply to the law of physics, but the latter will do it in its own specific way because it is both a physical object and an historical object. It answers to a stimulus by combining a direct physical reaction and a behavioural reaction resulting from 3,5 billions years of chance/selection evolution and heredity in its lineage. What makes it a life form is precisely this combination. Whether species evolve, for instance, toward 


\section{ORIGINS}

complexity or simplicity, is partly contingent and partly adaptive, and life forms succeed in lasting and perpetuating, at the genetic level, by tinkering, kludging, and recycling, as opposed to any rational an a-historical design, let alone any standardized answer when facing ever different and variable environments.

And on top of all, life is not simply matter, but dynamic fluxes of matter. At the cell or organism scale, material components come and go through metabolism, so that individuality itself can be questioned, because it only persist over time through extensive if not complete renewal of the parts, like ships of Theseus, constantly replaced piece by piece. Living entities keep their individuality through constant changes, and, as already foreseen by Immanuel Kant, can turn a part of their own living matter into new individuals [48]. At the evolutionary time scale, genetic sequences change, some being forgotten, some being introduced, some being expelled, and it will be all the same for any rational module on any streamlined chassis, or even unnatural nucleotides.

To summarize these features, even if little simplistic, one could say that life is an adaptive and dynamic change, and not order per se. That is not to say that some kind of biological order does not exist: it does and, obviously, biological organisms are also small systems, as Synthetic Biology sees them. But this dimension may only be the tip of the "lifeness iceberg" [49], and even its poorest account, precisely because so many mechanical systems are not living ones. Machines are finite and ordered objects that can be modified, unlike biological organisms that are changing entities that may reach some order. Engineering life to understand it presuppose that life is a given structure or pattern, whereas life is already itself a change, or a specific way to generate change on structures and patterns.

If accurate, this distinction has important consequences for Synthetic Biology. It would not at all debunk it, because exploring the mechanistic side of life still has a lot to bring. But it would implie that a rational engineering of life could at best question some its margins, even if spectacular. Developing new genetic chassis, or new cellular exosquelettons for life is more about harnessing it than understanding it, and would by contrast expose its elusive nature, not that it has any supernatural aspects, but just because we still have to invent a way to name it instead of using a shoehorn to turn it into a supercomplex machinery. As long as we will define life as a collection of things (classes of molecules, lists of function ${ }^{6}$, groups of cells, levels of organization) we can live with the illusion that engineering it is possible. If we accept life as a change itself, as a limit, a moving target, as a critical meeting point between disorder, history, plurality and matter, then it leads to at least two major consequences (i) it challenges the use of the concept of boundary (between living and non-living, between natural and synthetic, between parts, between modules, between levels) as a tool to understand the nature of life (ii) it may implies that "engineering life to better understand it", from an epistemological perspective, would therefore be unfounded.

\section{Synthetic Biology at the crossroads: Headlong rush or reboot?}

It would be a misunderstanding to conclude from the preceding section that Synthetic Biology is approaching an inescapable dead-end. By contrast with its high expectations, the discipline have also consistently promoted pragmatic, reflexive, and self-critical discussions, as a part of its own agenda since its (re)birth in 2004: most synthetic biologists are aware that having placed themselves under the spotlights, they need reactivity when facing an identity crisis. Some of these problems were for example addressed as soon as 2009, where five "hard truths" were listed: the lack of precision in defining of the "parts", the unpredictability of the circuitry, the unwieldy complexity, the incompatibility between parts, the threatening variability [50]. With the preceding section in mind, one may be entitled to think that most of these hurdles may hide even greater problems, for they only deal with precision or power.

\footnotetext{
${ }^{6}$ As done in note $d$ for clarification purposes.
} 
It is certainly not too late to step aside and adapt, and beside fundamental research, when considering the financial pressures, and the societal hopes and concerns, this may be necessary and urgent. Undoubtedly, this change is already happening with a new equilibrium between enthusiasm for a disruptive research and more achievable goals. Even for consolidated results (e.g. artemisinic acid production in yeast), the time gap between scientific demonstrations and industrial production can take years. This should lead to less long-term extraordinary claims and more classic biotechnology at work, given that the less complex a genetic module is, the more pre-existent genetic engineering methods can handle its accurate insertion and function in a given organism. In this resizing process, it will be a collective responsibility to make clear if the outcomes result from truly novel synthetic biology methods, or if they are just "biotechnology" redux, which would then mean that Synthetic Biology would have failed has a strategy.

But beside this quantitative and semantic issues, synthetic biologists have also started to take into account some of the above listed blind spots. Two examples can be highlighted. The first is the constitutive randomness of gene expression. Several reference authors in Synthetic Biology [51, 52] also have pioneered the field of stochastic gene expression [53, 54]. In a reductionist framework, such randomness is mainly a noise that drives cells away from the appropriate response, and the appropriate genetic circuitry to minimize such noise is crucial for rational design of genetic modules. Therefore, a detailed knowledge of the causes and consequence of such "noise" is of prime importance for Synthetic Biology, all the more than even deterministic circuits can generate unpredictable outcomes, such as bistable equilibria [55]. On the contrary, in probabilistic frameworks, stochasticity in gene expression may be the source of variation on which could rely exploratory and/or selective dynamics [56]. Therefore, progress in understanding this influence of randomness on cells and organisms will contribute to a large extent to redefine the actual balance of power between precision and chance at the source of biological order, and it is auspicious that specialists of biological randomness are involved in Synthetic Biology. The second example of a promising bridge with Synthetic Biology comes from ecology. Recent findings have underlined that instead of evolving exotic bacterial strains incorporating complex genetic modules, a promising strategy could be to coherently modify several strains by more classic engineering methods, they could depend on each other in a "synthetic consortium", in a more stable dynamic equilibrium [57]. In doing so, synthetic biologists would take advantage of the collective and ecosystemic nature of biological life instead of incurring it. Here genetic engineering may here be less disruptive or spectacular that many of the current DNA-based device constructions so far. But it may also be more sustainable and adaptable, at the strain scale as much as at the ecosystem scale. By merging novelty and efficiency, it may be a far more productive form of creativity.

These two examples show other directions ahead of biology than rational mechanization of its elementary units, without contravening to the methodological naturalist perspective whereby natural phenomenons must be explain by studying matter and its properties. For too long, biologists have equated matter and mechanism. By readdressing some of the most fundamental questions about the universal aspects of the living world, Synthetic Biology may have pushed this tendency farther than ever and started obtaining sometime spectacular results, but it has also been transparent enough to reveal its weaknesses, and even more, to reveal the implicit dead-ends toward which technologydriven approaches could lead biological science into. For a young discipline, that's already a lot to be credited for.

\section{Conclusion}

In quite a paradoxical move, Synthetic Biology has lead itself to a sometime self-contradictory situation where it can blur the boundary between what is synthetic and natural in the living world, and in the same time exalt its propension to product true novel synthetic entities. The latter claim, in addition to be a recast of an old play, would imply that human activities, how sophisticated they might be, are not gounded in nature. This demiurgical subtext is highly significant when it comes to analyse 


\section{ORIGINS}

the researchers' personal dreams of fame, but less convincing when it applies to its primary objects, which are the biological organisms supposed to be engineered. But still today, weaknesses of Synthetic Biology are more often attributed to a transitory lack of control, or interfacing, rather than on misleading preconceptions regarding its theoretical foundations. We have tried to identify such blind spots: in doing so we could not put emphasis enough on the fact that most of them are obviously well known by synthetic biologists themselves. But such a discipline is now more than a scientific field. For better or worse, it has become a social object, with social actors having contradictory expectations that may interfere with the scientific process stricto sensu. Figuring out that life is more about continuums than about thresholds [58-60] has major consequences. From a theoretical point of view, it would imply that creating synthetic life ${ }^{7}$, is an erroneous depiction of what this science does. Such statement would also impact applied research agendas. When, industry and research team up to develop synthetic organisms addressing a given medical or environmental issue, the former expects actual synthetic vectors or cells from the latter, and not discourses about blurry frontiers, transient constructions, and unpredictable evolution. This is why Synthetic Biology, in this ever challenging and competitive context, may face the peril of dilute itself in too much storytelling or self-fulfilling prophecies. But its resources are large, the first of which being its methodological creativity and its lack of inhibition when facing old biological constraints. So far, synthetic biologists have brought many proofs of principles that unexpected avenues can be taken by living beings, which is an inestimable gift for every biologists. For the next steps, should it strengthen its basis by reconnecting with founding principles in biology more than defying them, should it widen its ambitions by exploring with new eyes the various creative dynamics of biological life itself, Synthetic Biology would then undergo a consistent change and escape the naive illusions of technological normativity. It could very well then actually open a new era in life science.

\section{References}

[1] M. Shelley, Frankenstein or The Modern Prometheus (Lackington, Hughes, Harding, Mavor \& Jones, London, 1818)

[2] J.R. Karr, J.C. Sanghvi, D.N. Macklin, M.V. Gutschow, J.M. Jacobs, B. Bolival, Jr., N. AssadGarcia, J.I. Glass, M.W. Covert, Cell 150 (2012)

[3] M. Porcar, A. Danchin, V. de Lorenzo, V.A. dos Santos, N. Krasnogor, S. Rasmussen, A. Moya, Syst. Synth. Biol. 5 (2011)

[4] S.A. Benner, A.M. Sismour, Nature Reviews Genetics 6 (2005)

[5] L. Campos in M. Schmidt, A. Kelle, A. Ganguli-Mitra, H. de Vriend (Eds.) Synthetic Biology, the technoscience and its societal consequences (Springer, 2009)

[6] S. Leduc, La Biologie Synthétique (A. Poinat, 1912)

[7] W. Szybalski in A. Kohn and A. Shatkay (Eds.), Control of Gene Expression (Plenum Press, New York, 1974)

[8] M.A. O’Malley, A. Powell, J.F. Davies, J. Calvert, BioEssays 30 (2008)

[9] D.G. Gibson, J.L. Glass, C. Lartigue, V.N. Noskov, R.Y. Chuang, M.A. Algire, G.A. Benders, M.G. Montague, L. Ma, M.M. Moodie, C. Merryman, S. Vashee, R. Krishnakumar, N. AssadGarcia, C. Andrews-Pfannkoch, E.A. Denisova, L. Young, Z.Q. Qi, T.H. Segall-Shapiro, C.H. Calvey, P.P. Parmar, C.A. Hutchison 3rd, H.O. Smith, J.C. Venter, Science 329 (2010)

[10] N. Annaluru, H. Muller, L.A. Mitchell, S. Ramalingam, G. Stracquadanio, S.M. Richardson, J.S. Dymond, Z. Kuang, L.Z. Scheifele, E.M. Cooper, Y. Cai, K. Zeller, N. Agmon, J.S. Han, M. Hadjithomas, J. Tullman, K. Caravelli, K. Cirelli, Z. Guo, V. London, A. Yeluru, S. Murugan, K. Kandavelou, N. Agier, G. Fischer, K. Yang, J.A. Martin, M. Bilgel, P. Bohutski, K.M. Boulier, B.J.

\footnotetext{
${ }^{7}$ http://www.jcvi.org/cms/press/press-releases/full-text/article/first-self-replicatingsynthetic-bacterial-cell-constructed-by-j-craig-venter-institute-researcher/home/
} 
Capaldo, J. Chang, K. Charoen, W.J. Choi, P. Deng, J.E. DiCarlo, J. Doong, J. Dunn, J.I. Feinberg, C. Fernandez, C.E. Floria, D. Gladowski, P. Hadidi, I. Ishizuka, J. Jabbari, C.Y.L. Lau, P.A. Lee, S. Li, D. Lin, M.E. Linder, J. Ling, J. Liu, J. Liu, M. London, H. Ma, J. Mao, J.E. McDade, A. McMillan, A.M. Moore, W.C. Oh, Y. Ouyang, R. Patel, M. Paul, L.C. Paulsen, J. Qiu, A. Rhee, M.G. Rubashkin, I.Y. Soh, N.E. Sotuyo, V. Srinivas, A. Suarez, A. Wong, R. Wong, W.R. Xie, Y. Xu, A.T. Yu, R. Koszul, J.S. Bader, J.D. Boeke, S. Chandrasegaran1, Science 344 (2014)

[11] R. Gil, F.J. Silva, J. Peretó, A. Moya, Microbiol. Mol. Biol. Rev. 68 (2004)

[12] A. Danchin, FEBS Lett. 586 (2012)

[13] D.C. Smolke, Nat. Biotechnol. 27 (2009)

[14] E. Andrianantoandro, S. Basu1, D.K. Karig, R. Weiss, Mol. Sys. Biol. 2 (2006)

[15] D. Endy, Nature 438 (2005)

[16] C.J. Delebecque, A. Lindner, P.A. Silver, F.A. Aldaye, Science 333 (2011)

[17] T.F. Knight, Mol. Sys. Biol. 1 (2005)

[18] M. Meyer, Journ. Peer Prod. 2 (2012)

[19] D.-K. Ro, E.M. Paradise, M. Ouellet, K.J. Fisher, K.L. Newman, J.M. Ndungu, K.A. Ho, R.A. Eachus, T.S. Ham, J. Kirby, M.C.Y. Chang, S.T. Withers, Y. Shiba, R. Sarpong, J.D. Keasling, Nature, 440 (2006)

[20] F.M. Szczebara, C. Chandelier, C. Villeret, A. Masurel, S. Bourot, C. Duport, S. Blanchard, A. Groisillier, E. Testet, P. Costaglioli, G. Cauet, E. Degryse, D. Balbuena, J. Winter, T. Achstetter, R. Spagnoli, D. Pompon, B. Dumas, Nat. Biotech. 21 (2003)

[21] M. Schmidt, Bioessays 32 (2010)

[22] S.A. Benner, Acc. Chem. Res. 37 (2004)

[23] D.A. Malyshev, K. Dhami, T. Lavergne, T. Chen, N. Dai, J.M. Foster, I.R. Corrêa Jr, F.E. Romesberg, Nature 509 (2014)

[24] J.M. Bacher, R.A. Hughes, J. Tze-Fei Wong, A.D. Ellington, Trends Ecol. Evol. 19 (2004)

[25] S. A Benner, A. Ricardo, M. A Carrigan, Curr. Op. Chem. Biol. 8 (2004)

[26] P. Marlière, Syst. Synth. Biol. 3 (2009)

[27] S. Rasmussen, M.A. Bedau, L. Chen, D. Deamer, D.C. Krakauer, N.H. Packard, P.F. Stadler, Protocells, Bridging Nonliving And Living Matter (Cambridge University Press, Cambridge, 2009)

[28] P. Forterre, C R Acad Sci III. 318 (1995)

[29] S.A. Benner, Astrobiology 10 (2010)

[30] PC. Davies, S.A Benner, C.E. Cleland, C.H. Lineweaver, C.P. McKay, F. Wolfe-Simon (Eds), Astrobiology 9 (2009)

[31] W. Weber, M. Fussenegger, Nat. Rev. Gen. 13 (2012)

[32] G.J. Cooper, P.J. Kitson, R. Winter, M. Zagnoni, D.L. Long,L. Cronin, Angew Chem Int Ed Engl. 50 (2011)

[33] R. Descartes, Discours de la Méthode, Livre Cinquième (Jan Maire, Leiden, 1637)

[34] J. Onffray de La Mettrie, L’Homme Machine (Elie Luzac, Fils, Leiden, 1648)

[35] W. Paley, Natural Theology (R. Faulder, London, 1802)

[36] L. Kay, Who Wrote The Book Of Life? (Stanford University Press, Stanford, 2000)

[37] M. Isalan, Nature 488 (2012)

[38] R. Service, Science 339 (2013)

[39] P. Rabinow, N. Rose, BioSocieties 1 (2006)

[40] N. Wiener, Cybernetics: Or Control and Communication in the Animal and the Machine (MIT Press, Cambridge, 1948)

[41] H.J. Link, Sci. Eng. Ethics 19 (2013)

[42] R.E. Cobb, N. Sun, H. Zhao, Methods 60 (2013)

[43] H.H.Wang, F.J. Isaacs, P.A. Carr, Z.Z Sun, G. Xu, C.R. Forest, G.M. Church, Nature 460 (2009) 


\section{ORIGINS}

[44] M. Kistler (ed.) New Perspectives on Reduction and Emergence in Physics, Biology and Psychology, Special issue of Synthese, 151 (2006)

[45] T. Heams, Mat. Struct. Comput. Sci. 24 (2014)

[46] J.J. Kupiec, Specul. Sci. Technol. 6 (1983)

[47] J.J. Kupiec, The Origins Of Individuals (Word Scientific, Singapore, 2009)

[48] I. Kant, Critik der Urtheilskraft (Lagarde \& Friederich, Berlin \& Libau, 1790)

[49] C. Malaterre, Biol. Philo. 25 (2010)

[50] R. Kwok, Nature 463 (2010)

[51] N. Nandagopal, M.B. Elowitz, Science 233 (2011)

[52] J.J. Collins, M. Maxon, A. Ellington, M. Fussenegger, R. Weiss, H. Sauro, Nature 509 (2014)

[53] M.B. Elowitz, A.J. Levine, E.D. Siggia, P.S. Swain, Science 297 (2002)

[54] M. Kaern, T.C. Elston, W.J Blake, J.J. Collins, Nat Rev Genet. 6 (2005)

[55] H.H. McAdams, A. Arkin, Trends Genet. 15 (1999)

[56] J.J. Kupiec, Mol. Gen. Genet. 255 (1997)

[57] K. Brenner, L. You, F.H. Arnold, Trends. Biotech. 26 (2008)

[58] J. Griesemer, E. Szathmàry, in S. Rasmussen, M.A. Bedau, L. Chen, D. Deamer, D.C. Krakauer, N.H. Packard, P.F. Stadler, Protocells, Bridging Nonliving And Living Matter (Cambridge University Press, Cambridge, 2009)

[59] P. Forterre, The ISME Journal 7 (2013)

[60] J. Tamames, R. Gil, A. Latorre, J. Peretó, F.J. Silva, A. Moya, BMC Evol. Biol. 7 (2007) 